\title{
Familial Periodic Paralysis
}

National Cancer Institute

\section{Source}

National Cancer Institute. Familial Periodic Paralysis. NCI Thesaurus. Code C84709.

A group of genetic neurological disorders caused by mutations in genes involved in the sodium and calcium channels in nerve cells. It is characterized by episodes of muscle paralysis in which the affected muscles become flaccid and the deep tendon reflexes disappear. Between the episodes the affected muscles usually work normally. 